\title{
Determination of the number of light neutrino species from single photon production at LEP
}

\section{L3 Collaboration}

M. Acciarri ${ }^{\text {aa }}$, O. Adriani ${ }^{\text {p }}$, M. Aguilar-Benitez ${ }^{\mathrm{z}}$, S. Ahlen ${ }^{\mathrm{k}}$, J. Alcaraz ${ }^{\mathrm{z}}$,

G. Alemanni v, J. Allaby ${ }^{\mathrm{q}}$, A. Aloisio ${ }^{\mathrm{ac}}$, M.G. Alviggi ${ }^{\mathrm{ac}}$, G. Ambrosi ${ }^{\mathrm{s}}$, H. Anderhub aw, V.P. Andreev ${ }^{\text {al }}$, T. Angelescu ${ }^{\text {m }}$, F. Anselmo ${ }^{\mathrm{i}}$, A. Arefiev ${ }^{\text {ab }}$, T. Azemoon ${ }^{\text {c }}$, T. Aziz ${ }^{\mathrm{j}}$, P. Bagnaia ${ }^{\mathrm{ak}}$, L. Baksay $^{\text {ar }}$, R.C. Ball ${ }^{\mathrm{c}}$, S. Banerjee ${ }^{\mathrm{j}}$, Sw. Banerjee ${ }^{j}, K$. Banicz ${ }^{\text {at }}$, A. Barczyk ${ }^{\text {aw,au }}$, R. Barillère ${ }^{\mathrm{q}}$, L. Barone ${ }^{\text {ak }}$, P. Bartalini v , A. Baschirotto ${ }^{\text {aa }}$, M. Basile ${ }^{\mathrm{i}}, \mathrm{R}$. Battiston ${ }^{\text {ah }}$, A. Bay ${ }^{\mathrm{v}}, \mathrm{F}$. Becattini ${ }^{\mathrm{p}}$, U. Becker ${ }^{\circ}$, F. Behner ${ }^{\text {aw }}$, J. Berdugo ${ }^{\mathrm{z}}$, P. Berges ${ }^{\circ}$, B. Bertucci ${ }^{\text {ah }}$, B.L. Betev ${ }^{\text {aw }}$, S. Bhattacharya ${ }^{j}$, M. Biasini ${ }^{\text {ah }}$, A. Biland ${ }^{\text {aw }}$, G.M. Bilei ${ }^{\text {ah }}$, R. Bizzarri ${ }^{\text {ak }}$, J.J. Blaising ${ }^{\text {d }, ~ S . C . ~ B l y t h ~}{ }^{\text {ai }}$, G.J. Bobbink ${ }^{\text {b }}$, R. Bock ${ }^{\text {a }}$, A. Böhm ${ }^{\text {a }}$, L. Boldizsar ${ }^{\mathrm{n}}$, B. Borgia ${ }^{\text {ak }}$, D. Bourilkov ${ }^{\text {aw }}$, M. Bourquin ${ }^{\mathrm{s}}$, D. Boutigny ${ }^{\mathrm{d}}$, S. Braccini ${ }^{\text {s, J.G. Branson }}{ }^{\text {an }}$, V. Brigljevic ${ }^{\text {aw }}$, I.C. Brock ${ }^{\text {ai }}$, A. Buffini ${ }^{\text {p }}$, A. Buijs ${ }^{\text {as }}$, J.D. Burger ${ }^{\circ}$, W.J. Burger ${ }^{\text {ah }}$, J. Busenitz ${ }^{\text {ar }}$, X.D. Cai ${ }^{\circ}$, M. Campanelli ${ }^{\text {aw }}$, M. Capell ${ }^{\circ}$, G. Cara Romeo ${ }^{\text {i }}$, G. Carlino ${ }^{\text {ac }}$, A.M. Cartacci ${ }^{\mathrm{p}}$, J. Casaus ${ }^{\mathrm{z}}$, G. Castellini ${ }^{\mathrm{p}}$, F. Cavallari ${ }^{\mathrm{ak}}$, N. Cavallo ${ }^{\mathrm{ac}}$, C. Cecchi ${ }^{\mathrm{s}}$, M. Cerrada ${ }^{\mathrm{z}}$, F. Cesaroni ${ }^{\text {w }}$, M. Chamizo ${ }^{\text {z }}$, Y.H. Chang ay, U.K. Chaturvedi ${ }^{\mathrm{r}}$, S.V. Chekanov ${ }^{\text {ae }}$, M. Chemarin ${ }^{y}$, A. Chen ${ }^{\text {ay }}$, G. Chen ${ }^{g}$, G.M. Chen ${ }^{g}$, H.F. Chen ${ }^{t}$, H.S. Chen ${ }^{g}$, M. Chen ${ }^{o}$, G. Chiefari ${ }^{\mathrm{ac}}$, C.Y. Chien ${ }^{\mathrm{e}}$, L. Cifarelli ${ }^{\mathrm{am}}$, F. Cindolo ${ }^{\mathrm{i}}, \mathrm{C}_{\text {. Civinini }}{ }^{\mathrm{p}}$, I. Clare ${ }^{o}$, R. Clare ${ }^{\circ}$, H.O. Cohn ${ }^{\text {af }}$, G. Coignet ${ }^{\mathrm{d}}$, A.P. Colijn ${ }^{\mathrm{b}}$, N. Colino ${ }^{\mathrm{z}}$, S. Costantini h, F. Cotorobai ${ }^{\text {m}}$, B. de la Cruz ${ }^{\text {z }}$, A. Csilling ${ }^{\text {n }}$, T.S. Dai ${ }^{\text {o }}$, R. D’Alessandro ${ }^{\mathrm{p}}$, R. de Asmundis ${ }^{\mathrm{ac}}$, A. Degré ${ }^{\mathrm{d}}$, K. Deiters ${ }^{\text {au }}$, P. Denes ${ }^{\text {aj }}$, F. DeNotaristefani ${ }^{\text {ak }}$, D. DiBitonto ${ }^{\text {ar }}, \mathrm{M}$. Diemoz ${ }^{\text {ak }}$, D. van Dierendonck ${ }^{\mathrm{b}}$, F. Di Lodovico aw, C. Dionisi ak, M. Dittmar ${ }^{\text {aw }}$, A. Dominguez ${ }^{\text {an }}$, A. Doria ${ }^{\text {ac }}$, M.T. Dova ${ }^{\text {r,1 }}$, E. Drago ${ }^{\text {ac }}$, D. Duchesneau ${ }^{\text {d }}$, P. Duinker ${ }^{\text {b }}$, I. Duran ${ }^{\text {ao }}$, S. Dutta ${ }^{j}$,

S. Easo ${ }^{\text {ah }}$, Yu. Efremenko af, H. El Mamouni ${ }^{\text {y }}$, A. Engler ai, F.J. Eppling ${ }^{\circ}$,

F.C. Erné ${ }^{\mathrm{b}}$, J.P. Ernenwein ${ }^{\mathrm{y}}$, P. Extermann ${ }^{\mathrm{s}}$, M. Fabre ${ }^{\mathrm{au}}, \mathrm{R}$. Faccini ${ }^{\mathrm{ak}}$, S. Falciano ${ }^{\text {ak }}$, A. Favara ${ }^{\mathrm{p}}$, J. Fay ${ }^{\mathrm{y}}$, O. Fedin ${ }^{\text {al }}$, M. Felcini ${ }^{\mathrm{aw}}$, B. Fenyi ${ }^{\text {ar }}$, T. Ferguson ${ }^{\text {ai }}$, F. Ferroni ${ }^{\text {ak }}$, H. Fesefeldt ${ }^{\text {a }}$, E. Fiandrini ah ${ }^{\text {, J.H. Field }}{ }^{\text {s, }}$ 
F. Filthaut ${ }^{\text {ai }}$, P.H. Fisher ${ }^{\circ}$, I. Fisk ${ }^{\text {an }}$, G. Forconi ${ }^{\circ}$, L. Fredj ${ }^{\text {s }}$, K. Freudenreich ${ }^{\text {aw }}$, C. Furetta ${ }^{\text {aa }}$, Yu. Galaktionov ${ }^{\text {ab,o }}$, S.N. Ganguli ${ }^{j}$, P. Garcia-Abia ${ }^{\text {f }}$, S.S. Gau ${ }^{1}$, S. Gentile ${ }^{\text {ak }}$, J. Gerald ${ }^{\mathrm{e}}$, N. Gheordanescu ${ }^{\mathrm{m}}$, S. Giagu ${ }^{\mathrm{ak}}, \mathrm{S}$. Goldfarb ${ }^{\mathrm{v}}$, J. Goldstein k , Z.F. Gong ', A. Gougas ${ }^{\mathrm{e}}$, G. Gratta ${ }^{\mathrm{ag}}$, M.W. Gruenewald ${ }^{\mathrm{h}}$,

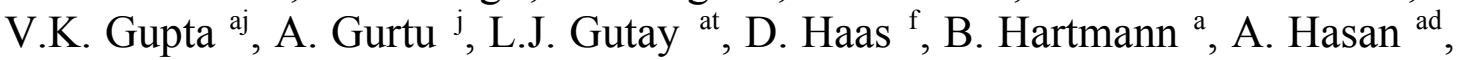
D. Hatzifotiadou i, T. Hebbeker h, A. Hervé q, J. Hirschfelder ai, W.C. van Hoek ${ }^{\text {ae }}$, H. Hofer ${ }^{\text {aw }}$, H. Hoorani ${ }^{\text {ai }}$, S.R. Hou ${ }^{\text {ay }}$, G. Hu ${ }^{\text {e, V. Innocente }}{ }^{\mathrm{q}}$, K. Jenkes ${ }^{\mathrm{a}}$, B.N. Jin ${ }^{\mathrm{g}}$, L.W. Jones ${ }^{\mathrm{c}}$, P. de Jong ${ }^{\mathrm{q}}$, I. Josa-Mutuberria ${ }^{\mathrm{z}}$, A. Kasser ${ }^{\mathrm{v}}$, R.A. Khan ${ }^{\text {r }}$, D. Kamrad ${ }^{\text {av }}$, Yu. Kamyshkov ${ }^{\text {af }}$, J.S. Kapustinsky ${ }^{x}$, Y. Karyotakis ${ }^{\text {d }}$, M. Kaur ${ }^{\text {r,2 }}$, M.N. Kienzle-Focacci ${ }^{\text {s }}$, D. Kim ${ }^{\text {ak }}$, D.H. Kim ${ }^{\text {aq }}$, J.K. Kim ${ }^{\text {aq }}$, S.C. Kim ${ }^{\text {aq }}$, W.W. Kinnison ${ }^{x}$, A. Kirkby ${ }^{a g}$, D. Kirkby ${ }^{\text {ag }}$, J. Kirkby ${ }^{\text {q }}$, D. Kiss ${ }^{\text {n, W. Kittel }}{ }^{\text {ae }}$, A. Klimentov ${ }^{\text {o,ab }}$, A.C. König ${ }^{\text {ae }}$, A. Kopp ${ }^{\text {av }}$, I. Korolko ${ }^{\text {ab }}$, V. Koutsenko ${ }^{\text {o,ab }}$, R.W. Kraemer ${ }^{\text {ai }}$, W. Krenz ${ }^{\text {a }}$, A. Kunin ${ }^{\text {o,ab }}$, P. Lacentre ${ }^{\text {av, }, \text {, }}$

P. Ladron de Guevara ${ }^{\mathrm{z}}$, G. Landi ${ }^{\mathrm{p}}$, C. Lapoint ${ }^{\circ}, \mathrm{K}$. Lassila-Perini ${ }^{\mathrm{aw}}$, P. Laurikainen ${ }^{\text {u }}$, A. Lavorato ${ }^{\text {am }}$, M. Lebeau ${ }^{\mathrm{q}}$, A. Lebedev ${ }^{\circ}$, P. Lebrun ${ }^{\mathrm{y}}$, P. Lecomte ${ }^{\text {aw }}$, P. Lecoq ${ }^{\text {q }}$, P. Le Coultre ${ }^{\text {aw }}$, H.J. Lee ${ }^{\text {h }}$, C. Leggett ${ }^{c}$, J.M. Le Goff ${ }^{\mathrm{q}}$, R. Leiste ${ }^{\text {av }}$, E. Leonardi ${ }^{\text {ak }}$, P. Levtchenko ${ }^{\text {al }}$, C. Li ${ }^{\mathrm{t}}$, C.H. Lin ${ }^{\text {ay }}$, W.T. Lin ${ }^{\text {ay }}$, F.L. Linde ${ }^{\text {b, }}$, L. Lista ${ }^{\text {ac }}$, Z.A. Liu ${ }^{\text {g }}$, W. Lohmann ${ }^{\text {av }}$, E. Longo ${ }^{\text {ak }}$, W. Lu ${ }^{\mathrm{ag}}$, Y.S. Lu ${ }^{\mathrm{g}}$, K. Lübelsmeyer ${ }^{\mathrm{a}}$, C. Luci ${ }^{\mathrm{ak}}$, D. Luckey ${ }^{\mathrm{o}}$, L. Luminari ${ }^{\mathrm{ak}}$, W. Lustermann ${ }^{\text {au }}$, W.G. Ma ${ }^{\text {t }}$, M. Maity ${ }^{j}$, G. Majumder ${ }^{j}$, L. Malgeri ${ }^{\text {ak }}$, A. Malinin ${ }^{a b}$, C. Maña ${ }^{z}$, D. Mangeol ae, S. Mangla j, P. Marchesini aw, A. Marin ${ }^{\text {k }}$, J.P. Martin ${ }^{\text {y }}$, F. Marzano ${ }^{\text {ak }}$, G.G.G. Massaro ${ }^{\text {b }}$, D. McNally ${ }^{\mathrm{q}}$, S. Mele ${ }^{\mathrm{q}}$, L. Merola $^{\text {ac }}$, M. Meschini ${ }^{\text {p }}$, W.J. Metzger ${ }^{\text {ae }}$, M. von der Mey ${ }^{\text {a }}$, Y. Mi ${ }^{\text {v }}$, D. Migani ${ }^{\mathrm{i}}$, A. Mihul ${ }^{\mathrm{m}}$, A.J.W. van Mil ${ }^{\text {ae }}$, H. Milcent ${ }^{\mathrm{q}}$, G. Mirabelli ${ }^{\text {ak }}$, J. Mnich ${ }^{\mathrm{q}}$, P. Molnar h , B. Monteleoni p, R. Moore c ${ }^{\text {, }}$ T. Moulik j, R. Mount ${ }^{\mathrm{ag}}$, F. Muheim ${ }^{\mathrm{s}}$, A.J.M. Muijs ${ }^{\text {b }}$, S. Nahn ${ }^{\circ}$, M. Napolitano ${ }^{\text {ac }}$, F. Nessi-Tedaldi ${ }^{\text {aw }}$, H. Newman ${ }^{\text {ag }}$,

T. Niessen ${ }^{\text {a }}$, A. Nippe ${ }^{\mathrm{v}}$, A. Nisati ${ }^{\text {ak }}$, H. Nowak ${ }^{\text {av }}$, Y.D. Oh ${ }^{\text {aq }}$, H. Opitz ${ }^{\text {a }}$, G. Organtini ${ }^{\text {ak }}$, R. Ostonen ", S. Palit ', C. Palomares ${ }^{\mathrm{z}}$, D. Pandoulas ${ }^{\mathrm{a}}$, S. Paoletti ${ }^{\text {ak }}$, P. Paolucci ac, H.K. Park ai ${ }^{\text {ai }}$ I.H. Park ${ }^{\text {aq }, ~ G . ~ P a s c a l e ~}{ }^{\text {ak }}$, G. Passaleva ${ }^{\text {q }}$, S. Patricelli ac, T. Paul ${ }^{1}$, M. Pauluzzi ${ }^{\text {ah }}$, C. Paus ${ }^{\text {q }}$, F. Pauss ${ }^{\text {aw }}$, D. Peach ${ }^{\text {q, }}$ Y.J. Pei a , S. Pensotti ${ }^{\text {aa }}$, D. Perret-Gallix ${ }^{\text {d }}$, B. Petersen ${ }^{\text {ae }}$, S. Petrak ${ }^{\text {h }}$, A. Pevsner ${ }^{\text {e, }}$ D. Piccolo ${ }^{\text {ac }}$, M. Pieri ${ }^{\text {p }}$, P.A. Piroué ${ }^{\text {aj }}$, E. Pistolesi ${ }^{\text {aa }}$, V. Plyaskin ${ }^{\text {ab }}$, M. Pohl aw , V. Pojidaev ab,p, H. Postema ${ }^{\circ}$, N. Produit ${ }^{\text {s }}$, D. Prokofiev ${ }^{\text {al }}$, J. Quartieri am, G. Rahal-Callot ${ }^{\text {aw }}$, N. Raja ${ }^{\text {j, P.G. Rancoita }}{ }^{\text {aa }}$, M. Rattaggi aa, G. Raven ${ }^{\text {an }}$, P. Razis ${ }^{\text {ad }}$, K. Read ${ }^{\text {af }}$, D. Ren ${ }^{\text {aw }}$, M. Rescigno ${ }^{\text {ak }}$, S. Reucroft ${ }^{1}$, T. van Rhee ${ }^{\text {as }}$, S. Riemann ${ }^{\text {av }}$, K. Riles ${ }^{c}$, O. Rind ${ }^{c}$, A. Robohm ${ }^{\text {aw }}$, J. Rodin ${ }^{\circ}$, B.P. Roe ${ }^{\text {c }}$, L. Romero ${ }^{\mathrm{z}}$, S. Rosier-Lees ${ }^{\mathrm{d}}$, Ph. Rosselet ${ }^{\mathrm{v}}$, W. van Rossum ${ }^{\text {as }}$, S. Roth a , J.A. Rubio ${ }^{\text {q }}$, D. Ruschmeier ${ }^{\text {h }}$, H. Rykaczewski ${ }^{\text {aw }}$, J. Salicio ${ }^{\text {q }}$, E. Sanchez ${ }^{\text {z }}$, M.P. Sanders ${ }^{\text {ae }}$, M.E. Sarakinos " , S. Sarkar j, G. Sauvage ${ }^{\text {d, }}$ 


\section{Schäfer ${ }^{\text {a }}$, V. Schegelsky ${ }^{\text {al }}$, S. Schmidt-Kaerst ${ }^{\text {a }}$, D. Schmitz ${ }^{\text {a }}$,} M. Schneegans ${ }^{\text {d }}$, N. Scholz ${ }^{\text {aw }}$, H. Schopper ${ }^{\text {ax }}$, D.J. Schotanus ${ }^{\text {ae }}$, J. Schwenke ${ }^{\text {a }}$,

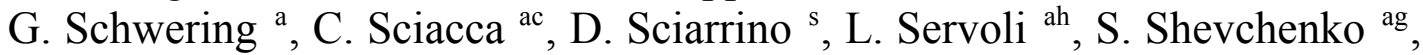
N. Shivarov ap, V. Shoutko ${ }^{\text {ab }}$, J. Shukla ${ }^{\mathrm{x}}$, E. Shumilov ${ }^{\mathrm{ab}}$, A. Shvorob ${ }^{\mathrm{ag}}$, T. Siedenburg ${ }^{a}$, D. Son ${ }^{\mathrm{aq}}$, V. Soulimov ${ }^{\text {ac }}$, B. Smith ${ }^{\circ}$, P. Spillantini ${ }^{\mathrm{p}}$, M. Steuer ${ }^{\circ}$, D.P. Stickland ${ }^{\text {aj }}$, H. Stone aj, B. Stoyanov ${ }^{\text {ap }}$, A. Straessner ${ }^{\text {a }}$, K. Sudhakar ${ }^{j}$, G. Sultanov ${ }^{r}$, L.Z. Sun ${ }^{\text {t }}$, G.F. Susinno ${ }^{\text {s }}$, H. Suter ${ }^{\text {aw }}$, J.D. Swain ${ }^{\mathrm{r}}$, X.W. Tang ${ }^{\mathrm{g}}$, L. Tauscher ${ }^{\mathrm{f}}$, L. Taylor ${ }^{1}$, Samuel C.C. Ting ${ }^{\mathrm{o}}$, S.M. Ting ${ }^{\circ}$, S.C. Tonwar ${ }^{\mathrm{j}}$, J. Tóth ${ }^{\mathrm{n}}$, C. Tully ${ }^{\text {aj }}$, H. Tuchscherer ${ }^{\text {ar }}$, K.L. Tung ${ }^{\mathrm{g}}$, Y. Uchida ${ }^{\circ}$, J. Ulbricht ${ }^{\text {aw }}$, U. Uwer ${ }^{\mathrm{q}}$, E. Valente ${ }^{\mathrm{ak}}$, G. Vesztergombi ${ }^{\mathrm{n}}$, I. Vetlitsky ${ }^{\text {ab }}$, G. Viertel ${ }^{\text {aw }}$, M. Vivargent ${ }^{\text {d }}$, S. Vlachos ${ }^{\text {f }}$, R. Völkert ${ }^{\text {av }}$, H. Vogel ${ }^{\text {ai }}$, H. Vogt ${ }^{\text {av }}$, I. Vorobiev ${ }^{\text {q,ab }}$, A.A. Vorobyov ${ }^{\text {al }}$, A. Vorvolakos ${ }^{\text {ad }}$, M. Wadhwa ${ }^{\text {f }}$, W. Wallraff ${ }^{\text {a }}$, J.C. Wang ${ }^{\circ}$, X.L. Wang ${ }^{\mathrm{t}}$, Z.M. Wang ${ }^{\mathrm{t}}$, A. Weber ${ }^{\mathrm{a}}$, S.X. Wu ${ }^{\circ}$, S. Wynhoff ${ }^{\text {a }}$, J. Xu ${ }^{\text {k }, ~ Z . Z . ~ X u ~}{ }^{\text {}}$, B.Z. Yang ${ }^{\text {t }}$, C.G. Yang ${ }^{\mathrm{g}}$, X.Y. Yao ${ }^{\mathrm{g}}$, J.B. Ye ${ }^{\mathrm{t}}$, S.C. Yeh ${ }^{\mathrm{az}}$, J.M. You ${ }^{\text {ai }}$, An. Zalite ${ }^{\text {al }}$, Yu. Zalite ${ }^{\text {al }}$,

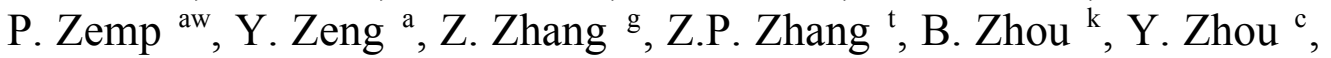
G.Y. Zhu ${ }^{\mathrm{g}}$, R.Y. Zhu ${ }^{\mathrm{ag}}$, A. Zichichi ${ }^{\mathrm{i}, \mathrm{q}, \mathrm{r}}$, F. Ziegler ${ }^{\text {av }}$

${ }^{a}$ I. Physikalisches Institut, RWTH, D-52056 Aachen, FRG ${ }^{4}$

III. Physikalisches Institut, RWTH, D-52056 Aachen, FRG ${ }^{4}$

${ }^{\mathrm{b}}$ National Institute for High Energy Physics, NIKHEF, and University of Amsterdam, NL-1009 DB Amsterdam, The Netherlands ${ }^{\mathrm{c}}$ University of Michigan, Ann Arbor, MI 48109, USA

${ }^{\mathrm{d}}$ Laboratoire d'Annecy-le-Vieux de Physique des Particules, LAPP,IN2P3-CNRS, BP 110, F-74941 Annecy-le-Vieux CEDEX, France

e Johns Hopkins University, Baltimore, MD 21218, USA

${ }^{\mathrm{f}}$ Institute of Physics, University of Basel, CH-4056 Basel, Switzerland

${ }^{\mathrm{g}}$ Institute of High Energy Physics, IHEP, 100039 Beijing, China ${ }^{5}$

h Humboldt University, D-10099 Berlin, FRG ${ }^{4}$

${ }^{\mathrm{i}}$ University of Bologna and INFN-Sezione di Bologna, I-40126 Bologna, Italy

${ }^{\mathrm{j}}$ Tata Institute of Fundamental Research, Bombay 400 005, India

${ }^{\mathrm{k}}$ Boston University, Boston, MA 02215, USA

${ }^{1}$ Northeastern University, Boston, MA 02115, USA

${ }^{\mathrm{m}}$ Institute of Atomic Physics and University of Bucharest, R-76900 Bucharest, Romania

${ }^{n}$ Central Research Institute for Physics of the Hungarian Academy of Sciences, H-1525 Budapest 114, Hungary ${ }^{6}$

${ }^{\circ}$ Massachusetts Institute of Technology, Cambridge, MA 02139, USA

${ }^{\mathrm{p}}$ INFN Sezione di Firenze and University of Florence, I-50125 Florence, Italy

${ }^{\mathrm{q}}$ European Laboratory for Particle Physics, CERN, CH-1211 Geneva 23, Switzerland

${ }^{\mathrm{r}}$ World Laboratory, FBLJA Project, CH-1211 Geneva 23, Switzerland

${ }^{\mathrm{s}}$ University of Geneva, CH-1211 Geneva 4, Switzerland

${ }^{\mathrm{t}}$ Chinese University of Science and Technology, USTC, Hefei, Anhui 230 029, China 5

" SEFT, Research Institute for High Energy Physics, P.O. Box 9, SF-00014 Helsinki, Finland

${ }^{v}$ University of Lausanne, CH-1015 Lausanne, Switzerland

${ }^{\mathrm{w}}$ INFN-Sezione di Lecce and Universitá Degli Studi di Lecce, I-73100 Lecce, Italy

${ }^{x}$ Los Alamos National Laboratory, Los Alamos, NM 87544, USA

${ }^{\text {y } I n s t i t u t ~ d e ~ P h y s i q u e ~ N u c l e ́ a i r e ~ d e ~ L y o n, ~ I N 2 P 3-C N R S, U n i v e r s i t e ́ ~ C l a u d e ~ B e r n a r d, ~ F-69622 ~ V i l l e u r b a n n e, ~ F r a n c e ~}$

${ }^{\mathrm{z}}$ Centro de Investigaciones Energeticas, Medioambientales y Tecnologicas, CIEMAT, E-28040 Madrid, Spain ${ }^{7}$

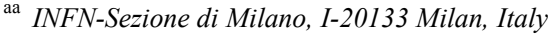

${ }^{\mathrm{ab}}$ Institute of Theoretical and Experimental Physics, ITEP, Moscow, Russia

ac INFN-Sezione di Napoli and University of Naples, I-80125 Naples, Italy

ad Department of Natural Sciences, University of Cyprus, Nicosia, Cyprus

ae University of Nijmegen and NIKHEF, NL-6525 ED Nijmegen, The Netherlands 
${ }^{\text {af }}$ Oak Ridge National Laboratory, Oak Ridge, TN 37831, USA

ag California Institute of Technology, Pasadena, CA 91125, USA

ah INFN-Sezione di Perugia and Universitá Degli Studi di Perugia, I-06100 Perugia, Italy

ai Carnegie Mellon University, Pittsburgh, PA 15213, USA

${ }^{\text {aj }}$ Princeton University, Princeton, NJ 08544, USA

ak INFN-Sezione di Roma and University of Rome, "La Sapienza", I-00185 Rome, Italy

${ }^{\text {al }}$ Nuclear Physics Institute, St. Petersburg, Russia

am University and INFN, Salerno, I-84100 Salerno, Italy

an University of California, San Diego, CA 92093, USA

${ }^{\text {ao }}$ Dept. de Fisica de Particulas Elementales, Univ. de Santiago, E-15706 Santiago de Compostela, Spain

ap Bulgarian Academy of Sciences, Central Lab. of Mechatronics and Instrumentation, BU-1113 Sofia, Bulgaria

${ }^{\text {aq }}$ Center for High Energy Physics, Korea Adv. Inst. of Sciences and Technology, 305-701 Taejon, South Korea

${ }^{\text {ar }}$ University of Alabama, Tuscaloosa, AL 35486, USA

${ }^{\text {as }}$ Utrecht University and NIKHEF, NL-3584 CB Utrecht, The Netherlands

at Purdue University, West Lafayette, IN 47907, USA

${ }^{\text {au }}$ Paul Scherrer Institut, PSI, CH-5232 Villigen, Switzerland

${ }^{\text {av }}$ DESY-Institut für Hochenergiephysik, D-15738 Zeuthen, FRG

${ }^{\text {aw }}$ Eidgenössische Technische Hochschule, ETH Zürich, CH-8093 Zürich, Switzerland

${ }^{\text {ax }}$ University of Hamburg, D-22761 Hamburg, FRG

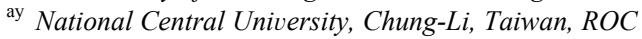

${ }^{a z}$ Department of Physics, National Tsing Hua University, Taiwan, ROC

Received 18 February 1998

Editor: K. Winter

\begin{abstract}
A determination of the number of light neutrino families performed by measuring the cross section of single photon production in $\mathrm{e}^{+} \mathrm{e}^{-}$collision near the $\mathrm{Z}$ resonance is reported. From an integrated luminosity of $100 \mathrm{pb}^{-1}$, collected during the years 1991-94, we have observed 2091 single photon candidates with an energy above $1 \mathrm{GeV}$ in the polar angular region $45^{\circ}<\theta_{\gamma}<135^{\circ}$. From a maximum likelihood fit to the single photon cross section, the $\mathrm{Z}$ decay width into invisible particles is measured to be $\Gamma_{\text {inv }}=498 \pm 12$ (stat) \pm 12 (sys) MeV. Using the Standard Model couplings of neutrinos to the Z, the number of light neutrino species is determined to be $N_{\nu}=2.98 \pm 0.07$ (stat) \pm 0.07 (sys). (C) 1998 Published by Elsevier Science B.V. All rights reserved.
\end{abstract}

\section{Introduction}

The determination of the number of light neutrino families, $N_{\nu}$, is one of the most fundamental results

\footnotetext{
${ }^{1}$ Also supported by CONICET and Universidad Nacional de La Plata, CC 67, 1900 La Plata, Argentina.

${ }^{2}$ Also supported by Panjab University, Chandigarh-160014, India.

${ }^{3}$ Supported by Deutscher Akademischer Austauschdienst.

${ }^{4}$ Supported by the German Bundesministerium für Bildung, Wissenschaft, Forschung und Technologie.

${ }^{5}$ Supported by the National Natural Science Foundation of China.

${ }^{6}$ Supported by the Hungarian OTKA fund under contract numbers T14459, T19181 and T24011.

${ }^{7}$ Supported also by the Comisión Interministerial de Ciencia y Technología.
}

obtained by the four LEP experiments. It has been derived from the measurement of the $\mathrm{Z}$ decays into light neutrinos, which form the invisible $\mathrm{Z}$ width, $\Gamma_{\text {inv }}$. In the Standard Model (SM) [1], the number of neutrino families is given by $N_{\nu}=\Gamma_{\text {inv }} / \Gamma_{\nu \nu}$ where $\Gamma_{\nu \nu}$ is the decay width of the $\mathrm{Z}$ into each neutrino family. The invisible width is also of interest because it is sensitive, in addition to the existence of further neutrino generations or any other pair of stable weakly interacting particles with mass less than $m_{\mathrm{Z}} / 2$, to possible processes outside the Standard Model, some of which are not currently accessible to experiments otherwise. Furthermore $\Gamma_{\text {inv }}$ is also sensitive to non-standard couplings of the known neutrinos to the $\mathrm{Z}$ and to phenomena such as the existence of right-handed neutrinos, mixing with the left- 
handed ones, which could lead to a non-integer $N_{\nu}$ [2]. Thus different, precise and complementary measurements are needed to address the above issues.

An indirect determination of $\Gamma_{\text {inv }}$ has been made at LEP through the analysis of the $\mathrm{Z}$ lineshape, subtracting the visible partial widths from the total one. The published result obtained by L3 in this approach is $N_{\nu}=2.98 \pm 0.05$ [3].

A direct measure of the $\mathrm{Z}$ invisible width, and thus of the number of light neutrino types, is based on the measurement of the cross section for the radiative process $\mathrm{e}^{+} \mathrm{e}^{-} \rightarrow \nu \bar{\nu} \gamma$ [4]. The signature of such events is a single photon from initial state radiation. Near the $\mathrm{Z}$ resonance, the cross section for this process is approximately proportional to $N_{\nu}$ since the contribution from $\mathrm{t}$-channel $\mathrm{W}$ exchange is small. This method is complementary in many respects to the indirect one. For instance in the indirect approach, exotic yet "visible" $\mathrm{Z}$ decays, not properly taken into account in the hadronic or in the leptonic selections, would give a contribution to $\Gamma_{\text {inv }}$ whereas they would not affect the present analysis.

Measurements of the cross section of the process $\mathrm{e}^{+} \mathrm{e}^{-} \rightarrow \nu \bar{\nu} \gamma$ have been performed at LEP [5-7] and at lower energies [8]. The single photon events have also been used to search for new phenomena [9]. The current world average on the number of neutrino families from the study of this reaction is $N_{\nu}=3.09$ \pm 0.13 [10]. In this paper we present a new measurement of the number of the neutrino families based on data collected with the L3 detector through the years 1992-94, corresponding to a total integrated luminosity of $90.3 \mathrm{pb}^{-1}$. The published 1991 data [6] are also used in the fit that gives the final number of neutrino families which is thus based on an overall luminosity of $100 \mathrm{pb}^{-1}$.

\section{Single photon trigger}

The L3 detector and its performance are described in detail in [11]. Concerning the hermeticity, the coverage is as follows: the polar angle acceptance of the BGO barrel electromagnetic calorimeter extends from $42.3^{\circ}$ to $137.7^{\circ}$; the BGO endcaps cover $11.4^{\circ}$ to $35.2^{\circ}$ and $144.8^{\circ}$ to $168.6^{\circ}$; the hadron calorimeter (HCAL) covers $6^{\circ}$ to $174^{\circ}$ and the muon spectrometer covers $36^{\circ}$ to $144^{\circ}$. The minimum angle at which particles are detected, critical for the suppression of
QED background, is defined by the luminosity monitors (LUMI). They cover the polar angular range $1.4^{\circ}<\theta<3.9^{\circ}$ on both sides of the interaction point. The region between the luminosity monitors and the hadron calorimeter endcaps is covered by two small lead rings instrumented with scintillator counters (ALR), leaving only a small region in between LUMI and ALR where particles can escape undetected.

Events with a low energy single photon in the BGO barrel are triggered by the first level energy trigger with a dedicated algorithm. The trigger is satisfied if an isolated energy deposit exceeding 1 $\mathrm{GeV}$ is found. Details of the trigger algorithm are described in [12].

The trigger efficiency is determined in two ways: from data and from a detailed simulation of the single photon trigger. The first method uses a sample of radiative Bhabha events with an isolated electron in the BGO barrel (the single electron control sample), which is triggered by requiring the coincidence of a charged track and an energy exceeding $30 \mathrm{GeV}$ in one of the luminosity monitors. The second one uses unbiased triggers as input of a dedicated simulation program $[5,6]$.

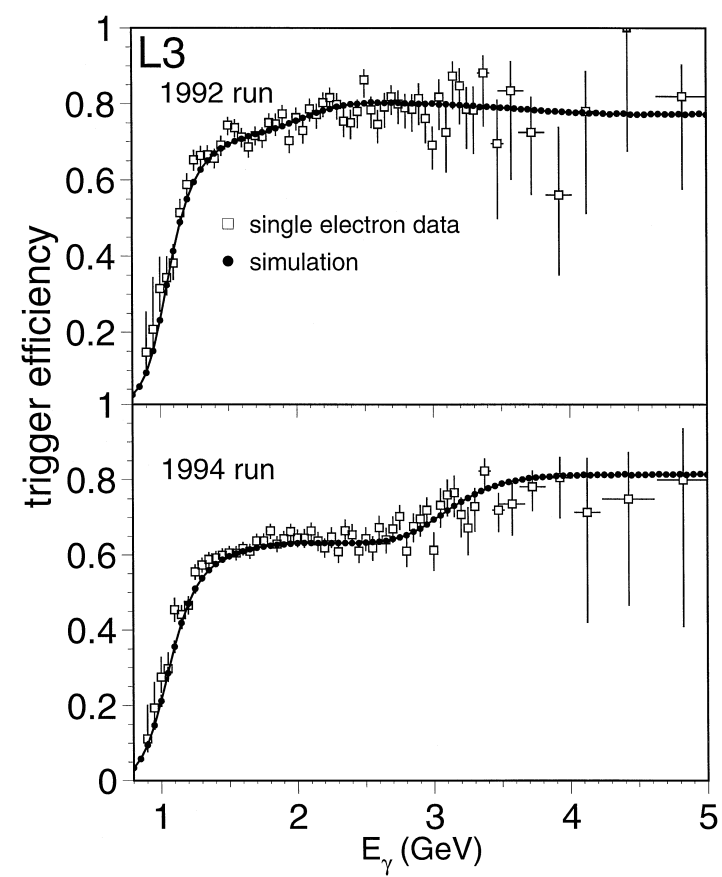

Fig. 1. The trigger efficiency as a function of the photon energy in 1992 and 1994. 
Fig. 1 shows the trigger efficiency as a function of the photon energy derived from the two methods for the 1992 and the 1994 data taking periods respectively. The agreement of the simulation with the single electron data at the level of $1 \%$, once folded with the single photon energy spectrum, justifies the use of the simulated curve also for periods where the statistics are limited.

\section{Event selection}

The experimental signature of the $\mathrm{e}^{+} \mathrm{e}^{-} \rightarrow \nu \bar{\nu} \gamma$ events is an electromagnetic shower and an otherwise "empty" detector as defined below.

The main sources of background are radiative QED processes, in which all other final-state particles, mainly produced at small polar angles, escape detection. Among these reactions, the dominant ones are radiative Bhabha scattering $\mathrm{e}^{+} \mathrm{e}^{-} \rightarrow \mathrm{e}^{+} \mathrm{e}^{-} \gamma$, the process $\mathrm{e}^{+} \mathrm{e}^{-} \rightarrow \gamma \gamma \gamma$, and the two-photon processes $\mathrm{e}^{+} \mathrm{e}^{-} \rightarrow \mathrm{e}^{+} \mathrm{e}^{-} \mathrm{X}$, where $\mathrm{X}$ is a $\pi^{0}, \eta, \eta^{\prime}, \mathrm{a}_{2}, \mathrm{f}$ or $l^{+} l^{-} \gamma$ [13]. A potential source of background events is also represented by cosmic muons. Due to the long integration time of the BGO ( $8 \mu \mathrm{s}$ starting $2.8 \mu \mathrm{s}$ before the beam crossing), an out of time cosmic muon emitting hard photon bremsstrahlung, when only the BGO is active, can fake a single photon event. This background is evaluated from the data, as described later.

In order to suppress contributions from these backgrounds, the following requirements are applied to the cluster found in the electromagnetic calorimeter:

1) an energy deposit in the $\mathrm{BGO}$ greater than $1 \mathrm{GeV}$ and less than $10 \mathrm{GeV}$, at a polar angle between $45^{\circ}$ and $135^{\circ}$, shared amongst at least five crystals;

2) the lateral shape of the energy deposit must be consistent with that expected from a single electromagnetic particle originating from the interaction point.

The detector is then required to be otherwise " "empty" as defined by the following criteria:

3) no other energy deposits in the BGO, consisting of 3 or more contiguous crystals and exceeding a total energy of $100 \mathrm{MeV}$;

4) no tracks in the central tracking chamber (TEC);
5) less than $1.5 \mathrm{GeV}$ deposited in either luminosity monitor;

6) no signal in the ALR;

7) less than $3 \mathrm{GeV}$ deposited in the HCAL;

8) no tracks measured in the muon spectrometer.

Requirement 3) reduces the contamination from two-photon production of resonances decaying into two or more photons; requirement 4) removes the single electron contamination and beam-gas or beam-wall events; requirements 5), 6) and 7) (in the regions not covered by the $\mathrm{BGO}$ ) reduce the $\mathrm{e}^{+} \mathrm{e}^{-} \gamma$ background. Requirements 2), 4), and 8) remove the contamination from the bremsstrahlung of cosmic rays.

The energy spectrum of the single photon candidates in the period 1992-94 is shown in Fig. 2 together with the Monte Carlo prediction for the signal expected from three light neutrino families and the backgrounds. The main background contribution is due to the $\mathrm{e}^{+} \mathrm{e}^{-} \gamma$ channel, when both electron and positron escape through the beam pipe $\left(\mathrm{E}_{\gamma}<1.5 \mathrm{GeV}\right)$ or one of the two leaves undetected between the ALR and the LUMI $\left(3.0 \mathrm{GeV}<\mathrm{E}_{\gamma}<\right.$

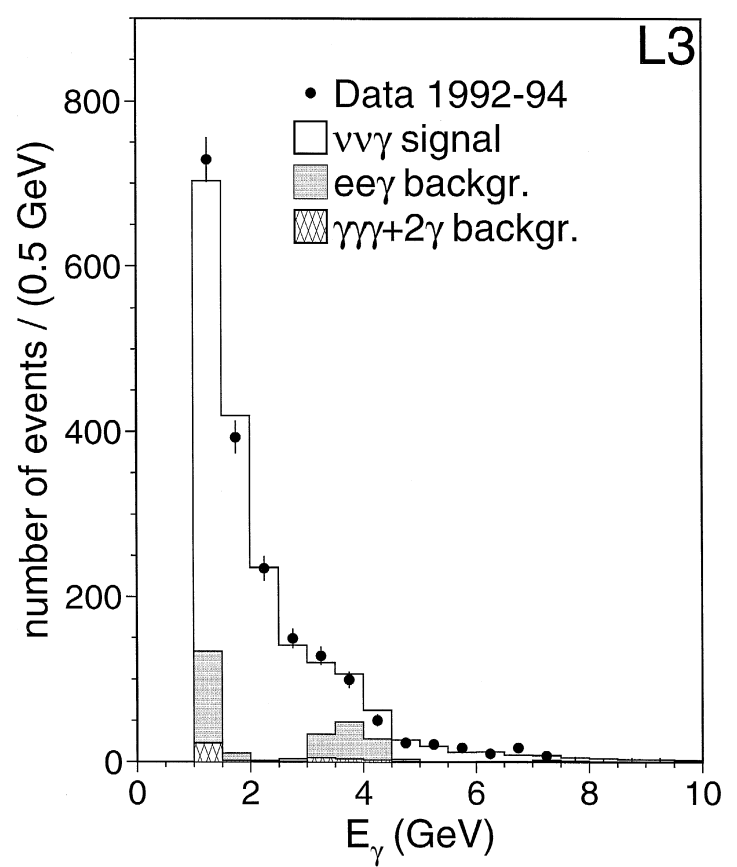

Fig. 2. The energy spectrum of the selected single photon candidates. The Monte Carlo predictions for the period 1992-94 are shown by different histograms. 
Table 1

Summary of the selected data sample and of the expected number of events. The 1991 data were the subject of our previous publication [6]

\begin{tabular}{|c|c|c|c|c|c|c|c|}
\hline \multirow{2}{*}{ Year } & \multirow{2}{*}{$\begin{array}{l}\sqrt{s} \\
(\mathrm{GeV})\end{array}$} & \multirow{2}{*}{$\begin{array}{l}\int \mathscr{L} \mathrm{d} t \\
\left(\mathrm{pb}^{-1}\right)\end{array}$} & \multirow{2}{*}{$\begin{array}{l}\text { Observed } \\
\text { events }\end{array}$} & \multicolumn{4}{|c|}{ Expected events } \\
\hline & & & & $\overline{N_{\bar{\nu} \gamma}}$ & $N_{\mathrm{e}^{+}} \mathrm{e}^{-} \gamma$ & $N_{\text {other back. }}$ & $\begin{array}{l}\text { Total } \\
\text { MC }\end{array}$ \\
\hline 1991 & $\begin{array}{l}88.56- \\
93.75\end{array}$ & 9.57 & 202 & 169.6 & 25.0 & 4.9 & 199.5 \\
\hline 1992 & 91.34 & 20.52 & 456 & 381.3 & 60.1 & 9.0 & 450.4 \\
\hline 1993 & 91.32 & 4.12 & 99 & 74.8 & 10.8 & 2.0 & 87.6 \\
\hline 1993 & 89.45 & 8.25 & 77 & 46.5 & 19.6 & 3.9 & 70.0 \\
\hline 1993 & 91.21 & 9.25 & 180 & 152.4 & 23.6 & 4.3 & 180.3 \\
\hline 1993 & 93.04 & 8.30 & 375 & 370.7 & 20.9 & 3.8 & 395.4 \\
\hline 1994 & 91.22 & 39.88 & 702 & 596.1 & 93.8 & 16.9 & 706.8 \\
\hline Total & & 99.89 & 2091 & 1791.4 & 253.8 & 44.8 & 2090.0 \\
\hline
\end{tabular}

$4.5 \mathrm{GeV}$ ) while the other stays in the beam pipe. Smaller sources of remaining backgrounds are $\gamma \gamma \gamma$ events and two-photon produced resonances.

In Table 1 we report the summary of the selected data sample along with the number of expected signal events for $N_{\nu}=3$, the background coming from the radiative Bhabha and the other minor backgrounds for the six sub-period samples defined for the years 1992-94. The number of events for 1991 data are taken from our previous publication [6].

\section{Systematic errors}

The main sources of systematic errors on the cross section are evaluated by performing the fit, described in the next section, with the parameters changed according to their maximum variation. They are summarised in what follows.

Trigger efficiency: the systematic error on the trigger efficiency is evaluated by varying the parameters entering in the trigger simulation, like calibration constants of the trigger channels, their resolution and the conversion factor with respect to the energy of the photon. The curves obtained for several choices of the parameters are convoluted with the single photon spectrum. From this we estimate a $1.2 \%$ of systematic uncertainty on the trigger efficiency. This estimate is confirmed by the single photon trigger efficiency measured using the single electron sample.

Background subtraction: the background mainly comes from the process $\mathrm{e}^{+} \mathrm{e}^{-} \rightarrow \mathrm{e}^{+} \mathrm{e}^{-} \gamma$. It is simulated by the Monte Carlo generator described in Ref. [14]. It is a first order generator that can be used in two ways exploiting the same matrix element: a single photon configuration, where the photon is in the barrel region and the two electrons stay at small angle, hence describing background events, and a single electron configuration where one electron is at large angle while the other two particles remain at small angles, hitting one of the two luminosity monitors (single electron sample). The cross section of the latter process is more than one order of magnitude larger than the single photon configuration and these events are used to study the accuracy of the Monte Carlo generator to reproduce the data. The single electron event selection is the same as the single photon one, once we replace requirement 4) by demanding a TEC track and requirement 5) by asking for at least $30 \mathrm{GeV}$ in one of the luminosity monitors. Fig. 3 shows for this sample the cosine of the polar angle of the charged tracks in the barrel multiplied by the sign of their charges. The data are compared with the expectation from the processes

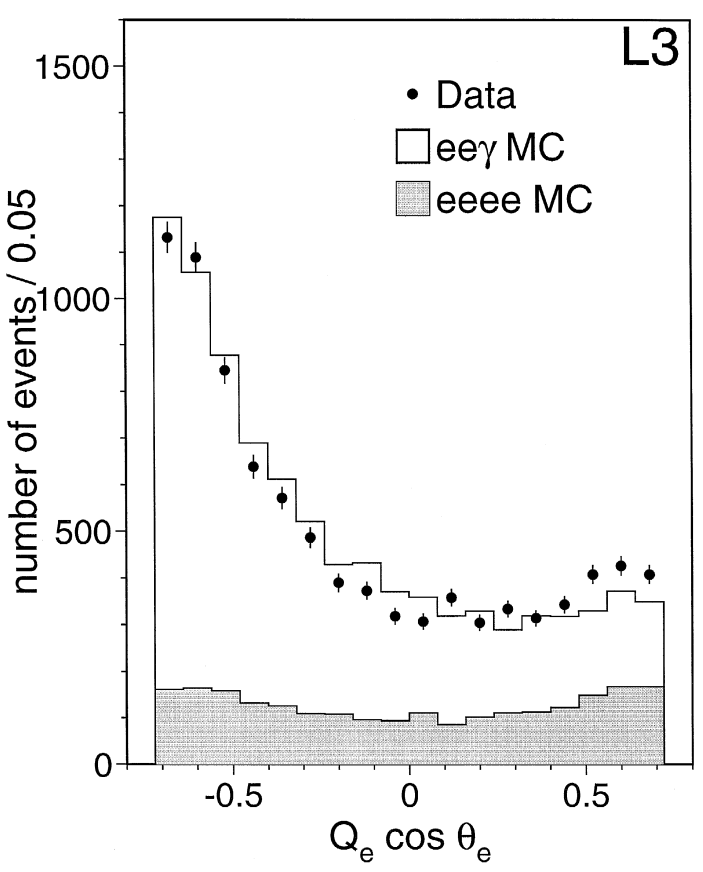

Fig. 3. The cosine of the single electron detected in the BGO barrel multiplied by the sign of its charge, $Q_{e}$. 
$\mathrm{e}^{+} \mathrm{e}^{-} \rightarrow \mathrm{e}^{+} \mathrm{e}^{-} \gamma$ and $\mathrm{e}^{+} \mathrm{e}^{-} \rightarrow \mathrm{e}^{+} \mathrm{e}^{-} \mathrm{e}^{+} \mathrm{e}^{-}$[15]. The electrons from radiative Bhabha events are preferentially scattered at low angles, while the electrons coming from the four electron final state have a flat angular distribution. The data are well reproduced by the two Monte Carlos justifying the use of the Bhabha Monte Carlo in the background subtraction. The difference in normalisation between data and Monte Carlo amounts to $(1.7 \pm 0.2) \%$.

A more stringent test is given by the analysis of the single photon - single tag events, that is a single photon in the barrel plus a signal with more than 30 $\mathrm{GeV}$ in one of the luminosity monitors, which is close to the background events where no signal in the luminosity monitors is required. The cross section for this process, which is only triggered by the single photon trigger, is approximately $20 \mathrm{pb}$. By comparing the data with the expected events from the processes $\mathrm{e}^{+} \mathrm{e}^{-} \rightarrow \mathrm{e}^{+} \mathrm{e}^{-} \gamma, \mathrm{e}^{+} \mathrm{e}^{-} \rightarrow \gamma \gamma \gamma$ [16], and the two-photon ones we estimate an error in the background subtraction of $6 \%$. It takes into account the Monte Carlo generator accuracy, the trigger efficiency and the detector simulation. The largest contribution comes from the position of the luminosity monitors which has to be reproduced as accurately as possible in the Monte Carlo description of the detector setup.

Selection efficiency: the selection described in the previous section is based on two main sets of requirements: the electromagnetic and the veto ones.

The efficiency to select electromagnetic showers, studied with a single electron sample obtained relaxing requirement 2), is $96.1 \%$ for the data and $98.5 \%$ for the Monte Carlo. This difference is due to inefficient crystals present in the data and not in the simulation and to a not accurate enough description of the lateral shower profile of low energy photons. To correct this effect the Monte Carlo events are weighted by the ratio of the two efficiencies. By changing the single electron sample used and by varying the electromagnetic requirements around the nominal ones we estimate an error of $0.5 \%$ on the correction factor.

The efficiency of the veto requirements is measured studying unbiased trigger events, which give the level of noise in the detector. It is determined for the individual data taking periods, and the average is $96.0 \%$. By changing the veto requirements around the nominal ones, we estimate an error on the veto efficiency of $0.5 \%$.

The selection efficiency for single photon events, measured by applying all requirements to a single photon Monte Carlo sample, is $(92.1 \pm 0.3) \%$ within the phase space defined by requirement 1 ). The error is dominated by the statistics of the sample used. The total error on the selection efficiency is $0.8 \%$.

Energy scale: the error on the energy scale is estimated by comparing the mass of the $\pi^{0}$ and $\eta$ measured in hadronic events [17], with their standard values [10]. The energy range of these photons is the same as the one of the single photon events studied. The error on the energy scale is $0.8 \%$.

Monte Carlo generators: we use for the signal the Monte Carlo generator NNGSTR described in [18]. It takes into account the complete second order diagrams describing the process, including electroweak corrections. The cross section is compared with an analytical calculation based on the structure function approach [19]. This yields an error on the predicted cross section of $0.7 \%$.

Cosmic ray background: to estimate the possible cosmic ray contamination a sample of potential single photon events produced by out of time cosmic muons is selected. The selection is based on TDCs of the scintillator counters which have a gate of $10 \mu \mathrm{s}$. In addition the event timing is inferred by the ratio of the photon energy measured by the fast trigger ADC with respect to the digital readout reconstructed offline. Due to the different integration time of the two ADCs, the ratio is equal to one for events in time with the beam crossing and it is less than one otherwise. In case the scintillators and the above ratio give consistent values the event is selected as an out of time cosmic. As a cross check we applied the same selection to the single electron control sample. No events were selected as out of time cosmic candidates.

We applied the requirements 2), 4) and 8) to the out of time cosmic sample. No events of the sample survive the selection requirements. We extrapolate this result to the single photon sample, obtaining a contamination of at most $0.25 \%$.

Luminosity and $\Gamma_{\nu \bar{\nu}}$ error:

The error on the luminosity measurement has been improved during the years going from $1 \%$ in 1991 , to $0.6 \%$ in 1992 and to less than $0.2 \%$ in 
Table 2

Breakdown of the systematic error contributions to $\Gamma_{\text {inv }}$ and $N_{\nu}$

\begin{tabular}{lll}
\hline Systematic error source & $\Delta \Gamma_{\text {inv }}(\mathrm{MeV})$ & $\Delta N_{\nu}$ \\
\hline Trigger efficiency & 8.4 & 0.050 \\
Background subtraction & 4.8 & 0.029 \\
Selection efficiency & 4.0 & 0.024 \\
Energy scale & 4.0 & 0.024 \\
Monte Carlo generators & 3.5 & 0.021 \\
Cosmic ray background & 1.7 & 0.010 \\
Luminosity error & 1.8 & 0.011 \\
$\Gamma_{\nu \nu}$ theoretical error & - & 0.004 \\
Fit procedure & 2.5 & 0.015 \\
Total error & 12.3 & \\
\hline
\end{tabular}

1993-94 [3,20]. By averaging these errors with the corresponding integrated luminosity we estimate an overall error of $0.37 \%$.

The computation of $\Gamma_{\nu \bar{v}}$ depends on electroweak corrections which are sensitive to $m_{\text {top }}$ and $m_{\text {Higgs }}$. With $m_{\text {top }}=175.6 \pm 5.5 \mathrm{GeV}[21]$ and $m_{\text {Higgs }}=300$ $\mathrm{GeV}$, varied between $69.5 \mathrm{GeV}$ [22] and $1000 \mathrm{GeV}$, we obtain $\Gamma_{\nu \bar{v}}=167.2 \pm 0.2 \mathrm{MeV}$. Errors due to other SM parameters are negligible.

Fit procedure: the experimental errors on $m_{\mathrm{Z}}, \Gamma_{\mathrm{Z}}$ and $\Gamma_{\mathrm{e}}$ [3], which are used in the fit described later, give the error on $\Gamma_{\text {inv }}$ and $N_{\nu}$ reported in Table 2 .

The contribution to $\Gamma_{\text {inv }}$ and $N_{\nu}$ of the various sources of systematic errors are summarised in Table 2.

\section{Results and conclusions}

The measured cross sections of the process $\mathrm{e}^{+} \mathrm{e}^{-}$ $\rightarrow \nu \bar{\nu} \gamma(\gamma)$, defined in the phase space volume $1 \mathrm{GeV}<\mathrm{E}_{\gamma}<10 \mathrm{GeV}$ and $45^{\circ}<\theta_{\gamma}<135^{\circ}$, are listed in Table 3. The total efficiency, including the trigger efficiency, is also given in Table 3. It takes also into account the inefficiency due to emission of additional photons. The measured cross sections are shown as a function of $\sqrt{s}$ in Fig. 4, where the cross sections measured at 7 different energies in 1991 are added after the rescaling to the 1992-94 phase space volume. The errors are statistical only and correspond to $68 \%$ C.L.

In the structure function approach [19] the cross section can be written as the convolution of a radia-
Table 3

Total efficiency and corrected cross section for $\mathrm{e}^{+} \mathrm{e}^{-} \rightarrow \nu \bar{\nu} \gamma(\gamma)$ at each center of mass energy

\begin{tabular}{llll}
\hline Year & $\sqrt{s}(\mathrm{GeV})$ & Efficiency & $\sigma(\mathrm{pb})$ \\
\hline 1992 & 91.34 & 0.572 & $32.9 \pm 1.8($ stat $) \pm 0.6($ sys $)$ \\
1993 & 91.32 & 0.594 & $35.2 \pm 4.1$ (stat) $\pm 0.6($ sys $)$ \\
1993 & 89.45 & 0.578 & $11.2 \pm 1.8$ (stat) \pm 0.3 (sys) \\
1993 & 91.21 & 0.570 & $28.8 \pm 2.5$ (stat) $\pm 0.5($ sys $)$ \\
1993 & 93.04 & 0.602 & $70.1 \pm 3.9$ (stat) \pm 1.1 (sys) \\
1994 & 91.22 & 0.505 & $29.4 \pm 1.3$ (stat) \pm 0.5 (sys) \\
\hline
\end{tabular}

tor function with an effective cross section $\sigma_{0}(s)$, which can be expressed in terms of $\Gamma_{\text {inv }}$ :

$$
\begin{aligned}
\sigma_{0}(s)= & \frac{12 \pi}{m_{\mathrm{Z}}^{2}} \frac{s \Gamma_{\mathrm{e}} \Gamma_{\mathrm{inv}}}{\left(s-m_{\mathrm{Z}}^{2}\right)^{2}+s^{2} \Gamma_{\mathrm{Z}}^{2} / m_{\mathrm{Z}}^{2}} \\
& + \text { W exchange terms }
\end{aligned}
$$

where $m_{\mathrm{Z}}, \Gamma_{\mathrm{Z}}$, and $\Gamma_{\mathrm{e}}$ are our measured values [3], for the $\mathrm{Z}$ mass, the total width and the electron partial width, respectively. In this way, we can allow $\Gamma_{\text {inv }}$ to vary while keeping the total width fixed. We

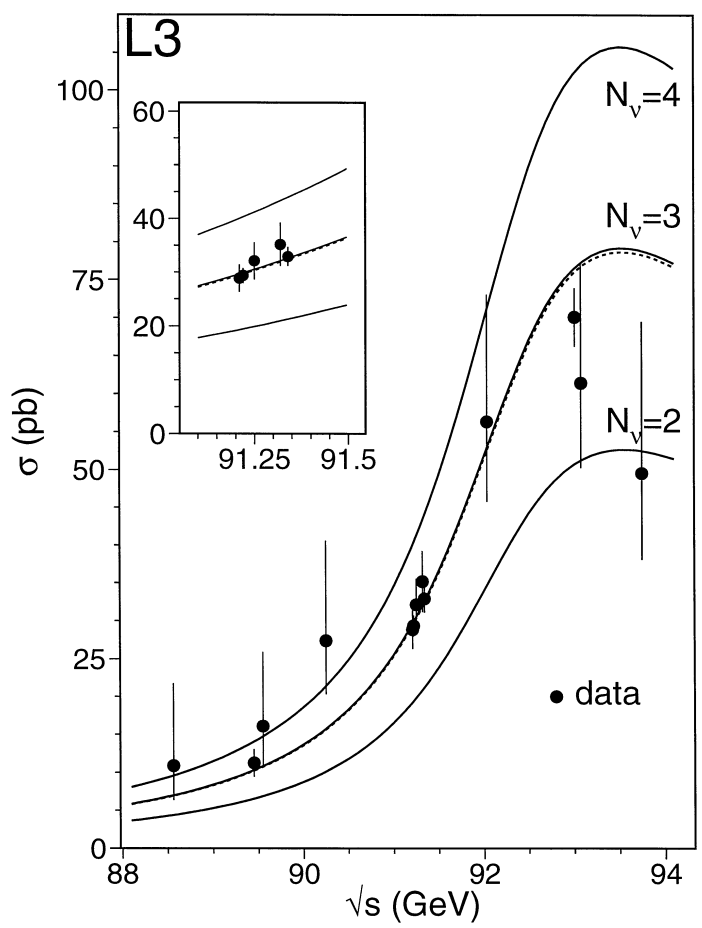

Fig. 4. The corrected single photon cross section $\left(45^{\circ}<\theta_{\gamma}<135^{\circ}\right.$ and $\left.\mathrm{E}_{\gamma}>1 \mathrm{GeV}\right)$ as a function of $\sqrt{s}$, compared with the prediction of Ref. [19]. Solid lines correspond to $N_{\nu}$ equal to two, three and four respectively, while the dashed line corresponds to the result of our fit. 
extract the invisible width by performing a maximum likelihood fit to the number of candidates shown in Table 1. We use Poisson probabilities calculated as a function of the expected number of signal events, which depends on $\Gamma_{\text {inv }}$, plus background events. The result of the overall fit to the 13 cross section measurements, along with the systematic errors discussed in the previous section and summarised in Table 2, yields:

$\Gamma_{\text {inv }}=498 \pm 12$ (stat) \pm 12 (sys) $\mathrm{MeV}$.

Assuming the Standard Model coupling of the neutrino pairs to the $\mathrm{Z}$, we determine the number of light neutrino families to be:

$N_{\nu}=2.98 \pm 0.07$ (stat) \pm 0.07 (sys)

This is in agreement with the L3 result from the line shape method [3]. It improves our previous results $[5,6]$ and the present world average on the number of light neutrino families determined with the single photon method [10].

\section{Acknowledgements}

We wish to express our gratitude to the CERN accelerator divisions for the excellent performance of the LEP machine. We acknowledge the contributions of all the engineers and technicians who have participated in the construction and maintenance of this experiment.

\section{References}

[1] S.L. Glashow, Nucl. Phys. 22 (1961) 579; S. Weinberg, Phys. Rev. Lett. 19 (1967) 1264; A. Salam, Elementary Particle Theory, N. Svartholm (Ed.), Stockholm, Almquist and Wiksell, 1968, p. 367.

[2] C. Jarlskog, Phys. Lett. B 241 (1990) 579.

[3] L3 Collaboration, M. Acciarri et al., Z. Phys. C 62 (1994) 551.

[4] A.D. Dolgov, L.B. Okun, V.I. Zakharov, Nucl. Phys. B 41 (1972) 197; E. Ma, J. Okada, Phys. Rev. Lett 41 (1978) 287; K.J. Gaemers, R. Gastmans, F.M. Renard, Phys. Rev. D 19 (1979) 1605; G. Barbiellini, B. Richter, J. Siegrist, Phys. Lett. B 106 (1981) 414; M. Chen, C. Dionisi, M. Martinez, X. Tata, Phys. Rep. 159 (1988) 201; L. Trentadue et al., Z
Physics at LEP I, G. Altarelli et al. (Eds.), CERN Yellow Book, 1989, vol. 1, CERN 89-08, p. 129.

[5] L3 Collaboration, B. Adeva et al., Phys. Lett. B 275 (1992) 209.

[6] L3 Collaboration, O. Adriani et al., Phys. Lett. B 292 (1992) 463.

[7] ALEPH Collaboration, D. Buskulic et al., Phys. Lett. B 313 (1993) 520; DELPHI Collaboration, P. Abreu et al., Z. Phys. C 74 (1997) 577; OPAL Collaboration, R. Akers et al., Z. Phys. C 65 (1995) 47.

[8] MAC Collaboration, W.T. Ford et al., Phys. Rev. D 33 (1986) 3472; ASP Collaboration, C. Hearty et al., Phys. Rev. D 39 (1989) 3207; H. Wu, Ph.D. thesis, Universitat Hamburg, 1986, unpublished; CELLO Collaboration, H.J. Behrend et al., Phys. Lett. B 215 (1988) 186; VENUS Collaboration, K. Abe et al., Phys. Lett. B 232 (1989) 431.

[9] L3 Collaboration, O. Adriani et al., Phys. Lett. B 297 (1992) 469; L3 Collaboration, M. Acciarri et al., Phys. Lett. B 346 (1995) 190; L3 Collaboration, M. Acciarri et al., Phys. Lett. B 412 (1997) 201; ALEPH Collaboration, CERN-PPE/97122, and references therein, submitted to Phys. Lett. B; DELPHI Collaboration, CERN-PPE/97-107, and references therein, Submitted to Z. Physik C; OPAL Collaboration, CERN-PPE/97-132, and references therein, Submitted to Z. Physik C.

[10] Physical Review D Particles and Fields, 1 July 1996, Part I, vol. 54

[11] L3 Collaboration, B. Adeva et al., Nucl. Inst. Meth. A 289 (1990) 35; M. Chemarin et al., Nucl. Inst. Meth. A 349 (1994) 345; M. Acciarri et al., Nucl. Inst. Meth. A 351 (1994) 300; A. Adam et al., Nucl. Inst. Meth. A 383 (1996) 342 .

[12] R. Bizzarri et al., Nucl. Inst. Meth. A 317 (1992) 463; P. Bagnaia et al., Nucl. Inst. Meth. A 324 (1993) 101; A 344 (1994) 213.

[13] F.L. Linde, Ph.D. thesis, Rijksuniversiteit Leiden, 1988; V.M. Budnev et al., Phys. Rep. C 15 (1975) 181.

[14] D. Karlen, Nucl. Phys. B 289 (1987) 23.

[15] R. Kleiss et al., Phys. Lett. B 180 (1986) 400.

[16] F.A. Berends, R. Kleiss, Nucl. Phys. B 186 (1981) 22.

[17] L3 Collaboration, B. Adeva et al., Phys. Lett. B 259 (1991) 199; L3 Collaboration, M. Acciarri et al., Phys. Lett. B 328 (1994) 223; B 371 (1996) 126.

[18] C. Mana, M. Martinez, R. Miquel, Z. Phys. C 48 (1990) 309; F.A. Berends et al., Nucl. Phys. B 301 (1988) 583.

[19] O. Nicrosini, L. Trentadue, Nucl. Phys. B 318 (1989) 1.

[20] I.C. Brock et al., Nucl. Inst. Meth. A 381 (1996) 236.

[21] CDF Collaboration, F. Abe et al., Phys. Rev. Lett. 74 (1995) 2626; D0 Collaboration, S. Abachi et al., Phys. Rev. Lett. 74 (1995) 2632; We use the average top mass as presented by A. Yagil, Int. Europhysics Conference on High Energy Physics, Jerusalem, August 19-26, 1997 to appear in the proceedings.

[22] L3 Collaboration, M. Acciarri et al., Phys. Lett. B 411 (1997) 373. 